\title{
Computational design of ductile magnesium alloy anodes for magnesium ion batteries
}

\author{
Vincent, Smobin; Chang, Jin Hyun; García Lastra, Juan Maria
}

Published in:

Batteries and Supercaps

Link to article, DOI:

10.1002/batt.202000240

Publication date:

2021

Document Version

Peer reviewed version

Link back to DTU Orbit

Citation (APA):

Vincent, S., Chang, J. H., \& García Lastra, J. M. (2021). Computational design of ductile magnesium alloy anodes for magnesium ion batteries. Batteries and Supercaps, 4(3), 522-528.

https://doi.org/10.1002/batt.202000240

\section{General rights}

Copyright and moral rights for the publications made accessible in the public portal are retained by the authors and/or other copyright owners and it is a condition of accessing publications that users recognise and abide by the legal requirements associated with these rights.

- Users may download and print one copy of any publication from the public portal for the purpose of private study or research.

- You may not further distribute the material or use it for any profit-making activity or commercial gain

- You may freely distribute the URL identifying the publication in the public portal

If you believe that this document breaches copyright please contact us providing details, and we will remove access to the work immediately and investigate your claim. 


\section{Batteries}

\section{\& Supercaps}

\section{p Chemistry Europe}

European Chemical Societies Publishing

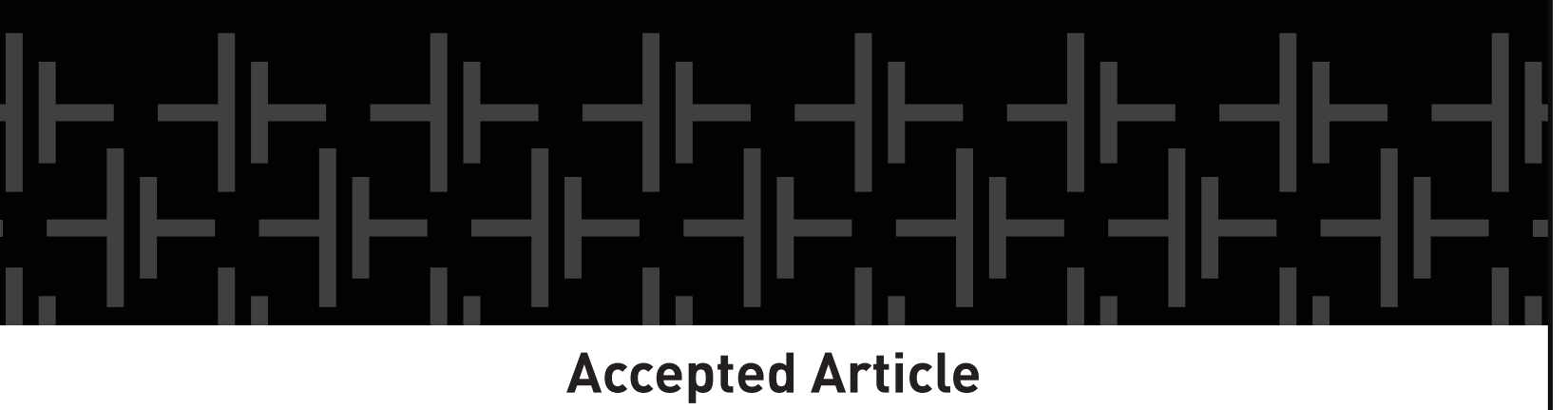

Title: Computational design of ductile magnesium alloy anodes for magnesium ion batteries

Authors: Smobin Vincent, Jin Hyun Chang, and Juan María García Lastra

This manuscript has been accepted after peer review and appears as an Accepted Article online prior to editing, proofing, and formal publication of the final Version of Record (VoR). This work is currently citable by using the Digital Object Identifier (DOI) given below. The VoR will be published online in Early View as soon as possible and may be different to this Accepted Article as a result of editing. Readers should obtain the VoR from the journal website shown below when it is published to ensure accuracy of information. The authors are responsible for the content of this Accepted Article.

To be cited as: Batteries \& Supercaps 10.1002/batt.202000240

Link to VoR: https://doi.org/10.1002/batt.202000240 


\title{
Computational design of ductile magnesium alloy anodes for magnesium batteries
}

\author{
Smobin Vincent, Jin Hyun Chang, and Juan Maria Garcia Lastra* \\ Department of Energy Conversion and Storage, Technical University of Denmark, DK-2800 Kgs. Lyngby, Denmark.
}

(Dated: December 3, 2020)

\begin{abstract}
The main advantage of $\mathrm{Mg}$ batteries over other metal counterparts is its ability to work with a pure metallic anode, achieving a very high specific capacity. Unfortunately, pure $\mathrm{Mg}$ is hard to machine due to its brittleness, making it extremely difficult to produce foils that are thin enough for practical battery applications. Alloying $\mathrm{Mg}$ with small amounts of doping elements can enhance its ductility. However, care should be given to ensure that the dopants do not interfere with the electrochemical process of plating and stripping of $\mathrm{Mg}$ from the anode during battery operation. Dopants should prefer to be in bulk or at a stacking fault rather than migrating to the surface to meet this requirement. In this work, we carried out a computational screening of 34 dopants that are reported to reduce $\mathrm{Mg}$ brittleness to check which of them energetically prefers to stay in bulk. We found that only 12 out of the 34 meet such a criterion. Y and Nd, two of the main dopants in the WE43 commercial alloys, are among the 12 beneficial doping elements, which presents a practical avenue for the exploration for superior $\mathrm{Mg}$ battery anode material.
\end{abstract}

\section{INTRODUCTION}

Rechargeable magnesium batteries (RMBs) are promising alternative to secondary rechargeable lithium batteries due to the relative abundance of magnesium compared to that of lithium. From the technical point of view, the main advantage of RMB is its ability to operate with a pure metallic anode since the plating and stripping of magnesium do not promote the formation of dendrites, unlike metallic lithium counterpart. Thanks to this property, RMBs display high volumetric capacity. However, one of the major obstacles in developing RMBs is the formation of a passivation layer at the magnesium metal anode surface in contact with conventional electrolytes such as $\mathrm{Mg}\left(\mathrm{BF}_{4}\right)_{2}$ or $\mathrm{Mg}\left(\mathrm{ClO}_{4}\right)_{2}$. The passivation layer hinders the diffusion of $\mathrm{Mg}$ ions and lowers the coulombic efficiency.[1] There are two proposed solutions to overcome the problems stemming from the passivation layer formation.

The first method is to use metals that form an alloy with magnesium as anode material. The magnesium still acts as a charge carrier that gets deposited on the anode upon charge, forming an alloy with the host anode metal. Several studies reported the use of $\mathrm{Bi}, \mathrm{Sn}, \mathrm{Sb}, \mathrm{In}$ and $\mathrm{Bi}-\mathrm{Sb}$ as an anode for RMB. [2-5] Even though reversible magnesiation is possible in these systems, they face some challenges such as capacity loss at high C-rates, sluggish Mg-ion kinetics and pulverisation of electrode due to volume expansion. $[1,6]$

The second route to suppress the passivation layer is to develop electrolytes alternative to the conventional $\mathrm{Mg}\left(\mathrm{BF}_{4}\right)_{2}$ and $\mathrm{Mg}\left(\mathrm{ClO}_{4}\right)_{2}$. Aurbach et al. reported a breakthrough by proposing organohaloaluminate-based electrolytes in ethereal solutions.[7] Several groups have followed up with the seminal work by Aurbach et al. to further develop new-generation electrolytes suitable for rechargeable RMBs. Some examples include the allphenyl complex (APC) electrolyte $\left((\mathrm{PhMgCl})_{2}-\mathrm{AlCl}_{3}\right.$ in tetrahydrofuran (THF)), [8] the magnesium aluminium chloride complex (MACC) electrolyte $\left(\mathrm{MgCl}_{2}-\mathrm{AlCl}_{3}\right.$ in dimethyl ether (DME)),[9], the $\mathrm{Mg}\left(\mathrm{CB}_{11} \mathrm{H}_{12}\right)_{2}$ salt in THF or DME[10] and the $\mathrm{Mg}\left[\mathrm{B}(\mathrm{HFIP})_{4}\right]_{2}$ salt in dyglime.[11]

Although developing a new electrolyte presents a promising avenue for future RMB research, magnesium also suffers from being too brittle. The brittle nature of magnesium is a serious obstacle to overcome in fabricating sufficiently thin foils to be used as RMB anodes. Consequently, magnesium anode's ductility has to be increased to develop practical RMBs in conjunction with next-generation electrolytes that suppress the formation of the passivation layer. Magnesium exhibits low ductility due to its hexagonal crystal structure. Existing experimental and theoretical studies indicate that doping magnesium with suitable elements will promote the formation of stacking fault, which in turn improves the ductility of magnesium.[12, 13] Stacking faults are crystal imperfections formed due to the addition or removal of an atomic layer. The excess energy per unit area due to the faults is known as stacking fault energy (SFE). There are three main types of basal stacking faults for $\mathrm{Mg}$ : one extrinsic stacking fault with $\mathrm{ABABCABAB}$ stacking sequence and two intrinsic stacking faults with $\mathrm{ABABCBCB}$ (called $I_{1}$ ) and $\mathrm{ABABCACA}$ (called $I_{2}$ ) stacking sequences. Sandlöbes et al. reported that reduction in the SFE of $I_{1}$ stacking fault $\left(I_{1} \mathrm{SF}\right)$ on doping improves the ductility of magnesium.[13]

Experimental reports on the use of doped $\mathrm{Mg}$ as $\mathrm{RMB}$ anode are relatively scarce. In aqueous electrolytes, Zhao et al.[14] and Wang et al.[15] have tested the AZ31 commercial alloys (Mg-3 wt\% Al-1 wt\% Zn). Wang et al. also tested AP65 (Mg-6 wt\% Al-5 wt\% Pb).[15] According to their reports, both AZ31 and AP65 exhibit larger overpotentials than pure Mg. Regarding non-aqueous electrolytes, Schloffer et al. studied Mg-1.55 wt\% Gd, Mg$1.63 \mathrm{wt} \% \mathrm{Zn}$ and $\mathrm{Mg}-1.02 \mathrm{wt} \% \mathrm{Gd}-1.01 \mathrm{wt} \% \mathrm{Zn}$ alloys with APC as electrolye.[16] They observed that after 5 cycles only the samples doped exclusively with $\mathrm{Zn}$ were showing a clear overpotential. Very recently, Mandai and Somekawa made a systematic analysis of $\mathrm{Mg}$ doped at 
0.3 wt\% with nine different elements, namely $\mathrm{Ag}, \mathrm{Al}, \mathrm{Bi}$, $\mathrm{Ca}, \mathrm{Li}, \mathrm{Mn}, \mathrm{Sn}, \mathrm{Y}$ and $\mathrm{Zn}$, with $\mathrm{Mg}\left[\mathrm{B}(\mathrm{HFIP})_{4}\right]_{2} /$ diglyme salt-electrolyte combination.[11] They reported that $\mathrm{Zn}$ and $\mathrm{Bi}$ increase the overpotential with respect to pure $\mathrm{Mg}$, while $\mathrm{Ag}$ and $\mathrm{Ca}$ decrease it. The other five elements (Al, Li, Mn, Sn and Y) did not seem to have any noticeable effect on the overpotential. The reason for the different influence of the dopants on the overpotentials remains unclear.

In this work, we hypothesize that the dopants that modify the electrochemical properties of $\mathrm{Mg}$, either benignly or detrimentally, are those that either segregate or migrate towards the surface of the system. By contrast, those dopants that stay at the bulk will be electrochemically inert (i.e., they will not affect the overpotentials, the electron conductivity at the surface, corrosion or passivation at the anode). Ideally, one should try to find dopants that enhance $\mathrm{Mg}$ ductility while concomitantly improve its electrochemical properties. The latter may be difficult to rationalize since several complex processes such as Mg diffusion at the surface and $\mathrm{Mg}$ solvation/disolvation need to be accounted, and they depend on the electrolyte being used. A more conservative approach is to focus solely on seeking dopants that reduce $\mathrm{Mg}$ brittleness without interfering with the electrochemical processes. Our strategy ensures to increase the ductility of RMB anode without sacrificing any other property.

Several authors have used density functional theory (DFT) to perform an exhaustive search to find suitable doping elements that improve the ductility of magnesium. $[12,13,17,18]$ The work of Zhang et al. has identified a set of 34 elements that improve the ductility of magnesium.[17] As an initial step of the investigation, we took these 34 elements and confirmed that they lower the SFE of $I_{1} \mathrm{SF}$ on alloying with magnesium based on our DFT calculations. As the dopants' impact on the electrochemical properties of the magnesium anode has not been explored, we investigated their propensity to migrate to the surface. Based on the propensity criterion, we have identified 12 dopants that could be added to magnesium to form a suitable ductile alloy anode while not compromising its electrochemical properties. Some of the identified systems can be readily purchased for further testing as they are commercially available.

This paper is structured in four sections. A short discussion on the brittleness of magnesium and how stacking faults helps to improve its ductility is presented in section 2. Section 3 covers the methodology and the computational details. The main results of this work are presented and discussed in section 4 . Some final comments are provided in the last section.

\section{DUCTILITY IN MAGNESIUM}

The brittleness of $\mathrm{Mg}$ originates from its insufficient number of independent slip systems needed to accommo- date deformations. According to the Von Mises criterion, a material requires five independent slip systems to allow deformations.[19] The possible slip systems in an hcp crystal, the crystal structure of magnesium at room temperature, are illustrated in Fig. 1. The corresponding number of independent slip systems are listed in Table I.

TABLE I. Independent slip systems in magnesium crystal system.[20]

\begin{tabular}{ccc}
\hline \hline Direction & Plane & Number of independent slip systems \\
\hline \multirow{3}{*}{$\langle a\rangle$} & Basal & 2 \\
& Prismatic & 2 \\
& Pyramidal-I & 4 \\
\hline \multirow{2}{*}{$\langle c+a\rangle$} & Pyramidal-I & 5 \\
& Pyramidal-II & 5 \\
\hline \hline
\end{tabular}

Basal and prismatic slips along $\langle a\rangle$ direction offer two independent slip systems each. The four slip systems of pyramidal-I along $\langle a\rangle$ direction is crystallographically equivalent to the combination of basal $\langle a\rangle$ and prismatic $\langle a\rangle$ slips.[20] Therefore, only four independent slip systems are attainable in $\langle a\rangle$ direction. On the other hand, pyramidal-I and pyramidal-II slips along $\langle c+a\rangle$ direction offer five independent slip systems, and activating any of the slips along $\langle c+a\rangle$ direction can satisfy the Von Mises criterion. Wu and Curtin showed that $\langle c+a\rangle$ dislocations are metastable even in pure $\mathrm{Mg}$ once they are formed, although the their formation is energetically unfavorable.[21] Therefore, finding dopants that activate and further stabilize the $\langle c+a\rangle$ dislocations is a suitable strategy to enhance the ductility of $\mathrm{Mg}$.

The dopants activate $\langle c+a\rangle$ dislocations through the promotion of stacking faults.[13] Sandlöbes et al. investigated the relationship between ductility and SFEs in $\mathrm{Mg}$ and $\mathrm{Mg}-\mathrm{Y}$ alloys.[13] They observed a reduction in $\mathrm{SFE}$ of $\mathrm{Mg}$ upon alloying with $\mathrm{Y}$ and reported that the improved ductility of $\mathrm{Mg}-\mathrm{Y}$ alloys was due to the high activity of pyramidal-I and pyramidal-II $\langle c+a\rangle$ dislocations. The plausible explanation for this is that $I_{1}$ stacking fault $\left(I_{1} \mathrm{SF}\right)$ can act as nucleation source for $\langle c+a\rangle$ dislocations.[12] Thus, dopants that reduce the SFE (relative to that of pure $\mathrm{Mg}$ ) for the $I_{1} \mathrm{SF}$ will promote $\langle c+a\rangle$ dislocations, and consequently will enhance $\mathrm{Mg}$ ductility. Therefore, we limit the scope of the stacking faults to $I_{1} \mathrm{SF}$ in this study.

\section{COMPUTATIONAL METHODS}

Three conditions need to be fulfilled in order to assess whether the addition of dopants is beneficial for RMB applications:

- The dopant should improve magnesium's ductility (the SFE for the $I_{1} \mathrm{SF}$ should be lowered after dopant addition). 

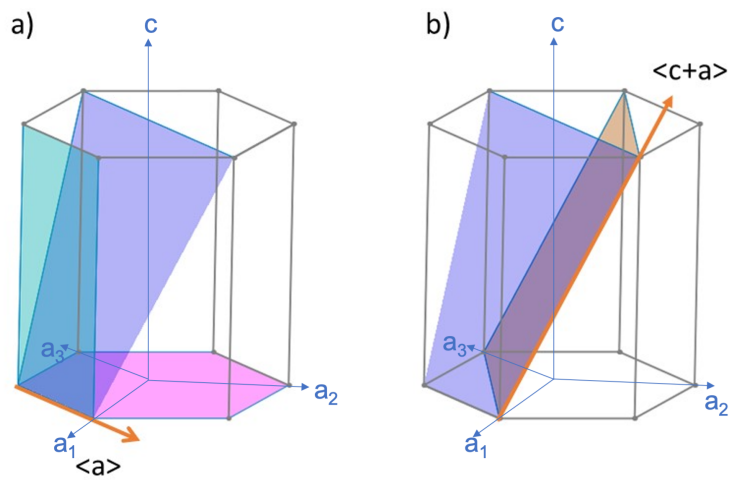

Basal

$\{10 \overline{1} 0\} \quad$ Prismatic

$\{10 \overline{1} 1\} \quad$ Pyramidal - I

$\{11 \overline{2} 2\} \quad$ Pyramidal - I

FIG. 1. Possible slip systems in hexagonal system. a) Basal, prismatic and pyramidal-I slip systems in $\langle a\rangle$ direction and b) pyramidal-I and pyramidal-II slip systems in $\langle c+a\rangle$ direction.

- The dopant has to form a thermodynamically stable alloy with magnesium.

- The dopant should be more stable either in bulk or at stacking faults than on the surface to form a dopant-free surface.

Regarding the first condition, the first-principle study of Zhang et al. on $I_{1} \mathrm{SF}$ identified 34 dopants that decrease the SFE of magnesium.[17] These elements are Al, $\mathrm{Ba}, \mathrm{Bi}, \mathrm{Ca}, \mathrm{Ce}, \mathrm{Cs}, \mathrm{Dy}, \mathrm{Er}, \mathrm{Eu}, \mathrm{Ga}, \mathrm{Gd}, \mathrm{Hf}, \mathrm{Ho}, \mathrm{In}, \mathrm{K}$, $\mathrm{La}, \mathrm{Lu}, \mathrm{Na}, \mathrm{Nd}, \mathrm{Pb}, \mathrm{Pr}, \mathrm{Rb}, \mathrm{Sc}, \mathrm{Sm}, \mathrm{Sn}, \mathrm{Sr}, \mathrm{Tb}, \mathrm{Ti}$, $\mathrm{Tl}, \mathrm{Tm}, \mathrm{Y}, \mathrm{Yb}, \mathrm{Zn}$ and $\mathrm{Zr}$. In our study, we restricted ourselves to this set of 34 dopants with a doping concentration of $\sim 2.08 \%$ for all alloy structures (i.e., one dopant atom per 48 atoms), which lies in the typical doping level of commercial alloys ( $\sim 1 \%$ to $3 \%)$. We carried out our own first-principle $I_{1} \mathrm{SF}$ study since the SFE depends on the doping level, while no SFEs have been reported for a $2.08 \%$ doping concentration to the best of our knowledge.

The SFE for the $I_{1} \mathrm{SF}$ was calculated using

$$
\mathrm{SFE}=\frac{E^{I_{1} \mathrm{SF}}-E^{\mathrm{bulk}}}{2 A}
$$

where $E^{I_{1} \mathrm{SF}}$ and $E^{\text {bulk }}$ are the energies of the structures with and without stacking faults, respectively. $A$ is the area of the stacking fault plane, which corresponds to the area of the XY plane in this work. The term "bulk" refers to the bulk of the material in a pristine form without stacking faults. The $I_{1} \mathrm{SF}$ and bulk structures used in the calculations are shown in Fig. 2. We tested the size of the SF supercell to make sure that the interactions between the SF defects are negligible. The change in SFE for the supercells with SFs seperated by more than 8 atomic layers is less than $1 \mathrm{~mJ} / \mathrm{m}^{2}$. To perform calculations with low doping concentration $(\sim 2.08 \%)$, we employed $2 \times 2 \times 12$ supercell of hcp containing 96 atoms for both structures, and two magnesium atoms were substituted with the dopants for the alloy structures. Due

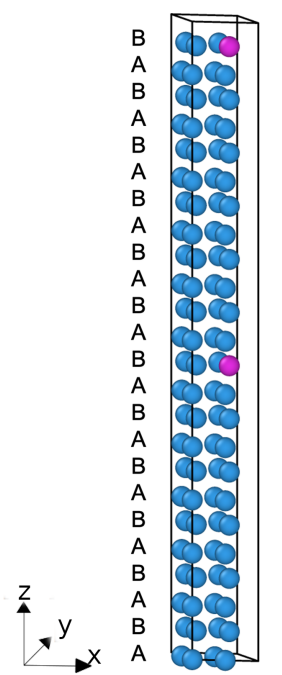

a) Pristine bulk

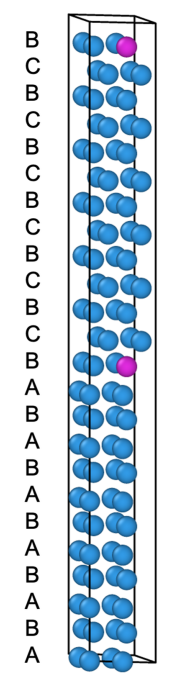

b) $l_{1}$ stacking fault
FIG. 2. Structures of a) pristine bulk and b) $I_{1} \mathrm{SF}$. Mg atoms are shown in blue and the dopants are shown in pink.

to periodic boundary conditions, the $I_{1} \mathrm{SF}$ structure has two stacking-fault planes separated by 12 atomic layers to minimize the interaction between them, and a dopant was placed on each of the stacking faults.

We used Vienna Ab initio Simulation Package (VASP) to perform first-principle calculations based on DFT.[22, 23] The Perdew-Burke-Ernzerhof (PBE) parameterized generalized-gradient approximation (GGA) functional was employed to describe the exchange and correlation effects,[24] and the projector augmented wave (PAW) method was used to account for the core-valence interaction. [25] A plane-wave basis set with cut-off energy of $520 \mathrm{eV}$ was used for all calculations. The Brillouin zone is integrated with the $k$-point mesh generated using the Monkhorst-Pack scheme with a $k$-point density of 4.5 per $\AA^{-1}$ for all the structures. The convergence of the $k$-point 
density was tested for magnesium and all other elements in this study, and the difference in total energy was found to be less than $2 \mathrm{meV} /$ atom. The convergence criterion for the electronic self-consistent loop was set to $10^{-6} \mathrm{eV}$, and the atomic positions were relaxed until the force on each atom reached below $10^{-2} \mathrm{eV}^{-1}$. The structure and atomic positions were relaxed for both bulk and $I_{1} \mathrm{SF}$ structures, and only the atomic positions were relaxed for the slabs.

The second and third conditions (the alloy stability and the dopant's propensity to migrate to the surface, respectively) were evaluated based on conventional expressions for formation energies. The formation energies of an alloy when a dopant is in bulk, at stacking fault or on the surfaces were calculated using

$$
E_{f}^{\text {struct }}=\frac{E^{\text {struct }}\left(\mathrm{Mg}_{m-n} \mathrm{X}_{n}\right)-E^{\text {struct }}\left(\mathrm{Mg}_{m}\right)-n E(\mathrm{X})+n E(\mathrm{Mg})}{n},
$$

where struct can be either bulk, $I_{1} \mathrm{SF}$, or surface terminations (e.g., (0001), (1010), and (1011)). $E^{\text {struct }}\left(\mathrm{Mg}_{m-n} \mathrm{X}_{n}\right)$ is the energy of the structure where $n$ out of $m \mathrm{Mg}$ atoms are substituted with dopant atoms. $E^{\text {struct }}\left(\mathrm{Mg}_{m}\right)$ is the energy of the same structure with no dopants (i.e., all $m$ atoms are $\mathrm{Mg}$ ). $E(\mathrm{X})$ is the energy per atom of $\mathrm{X}$ in its most stable crystalline structure. $E(\mathrm{Mg})$ is the energy per atom of $\mathrm{Mg}$ in hcp. All the simulations for calculating formation energies have the same calculation parameters used for the stacking fault simulations for consistency. In addition, the number of atoms in the supercells were kept to 96 for all the cases (i.e., $m=96$ in Eq. (2)), with two dopant atoms placed as far apart from each other as possible.

The stability of the alloy (second condition) was evaluated based on the formation energy with the dopant in the bulk/stacking fault. The $E_{f}^{\text {bulk }}$ or $E_{f}^{I_{1} \mathrm{SF}}$ should be negative to form a stable alloy. The dopants will segregate and form a separate phase if they are positive. The energy of the bulk and $I_{1} \mathrm{SF}$ structures were also computed using the structure shown in Fig. 2. The propensity of the dopant to migrate to the surface (third condition) was analyzed by comparing the formation energies of the alloys when the dopant is in the bulk and at stacking fault versus on surfaces. The relative energy of the dopant in the bulk and at the stacking fault versus on the surface are written as

$$
\Delta E^{\mathrm{bulk}}=E_{f}^{\mathrm{bulk}}-E_{f}^{\mathrm{surface}}
$$

and

$$
\Delta E^{I_{1} \mathrm{SF}}=E_{f}^{I_{1} \mathrm{SF}}-E_{f}^{\mathrm{surface}},
$$

respectively. The superscript 'surface' refers to one of

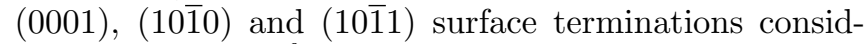
ered, and the $E_{f}^{\text {surface }}$ is for the most thermodynamically favorable $\mathrm{Mg}$ surface for the dopant. At least one of $\Delta E^{\text {bulk }}$ or $\Delta E^{I_{1} \mathrm{SF}}$ should be negative in order to ensure that the dopant does not migrate to the surface.

The preliminary step for assessing the migration of the dopants towards the surface is to determine which
$\mathrm{Mg}$ surface terminations are thermodynamically most favorable. The surfaces formed in magnesium at equilibrium condition were identified using the Wulff plot analysis. The 12 symmetrically distinct surfaces in magnesium crystal with the lowest Miller indices were considered for the Wulff construction. We followed the methodology in the work of Tran et al.[26] to obtain the Wulff plot using our lattice constants and simulation parameters. Tran et al. reported that the minimum thickness of $10 \AA$ for both slab and vacuum is sufficient to converge surface energies to within $0.02 \mathrm{~J} / \mathrm{m}^{2}$. We used slab structures with two surfaces separated by a minimum of 8 atomic layers $(>10 \AA)$ and vacuum thickness of $10 \AA$ for the surface energy calculations. The surface energy, $\gamma^{\text {surf }}$, of different surfaces required for obtaining the Wulff shape is calculated using

$$
\gamma^{\text {surf }}=\frac{E^{\text {slab }}-n^{\text {slab }} E_{\mathrm{Mg}}}{2 A^{\text {slab }}},
$$

where $E^{\text {slab }}$ is the energy of the slab model with the surface, $n^{\text {slab }}$ is the number of atoms in slab structure and $A^{\text {slab }}$ is the area of the slab along the surface plane. The Wulff shape was obtained using the Wulff analysis module implemented in Pymatgen.[26, 27] After identifying the surfaces formed in magnesium at equilibrium conditions, the formation energies of the surfaces were calculated using Eq. (2).

\section{RESULTS AND DISCUSSION}

\section{A. Ductility analysis}

The alloying elements that reduce SFE of magnesium upon alloying are identified as suitable dopants that enhance the ductility of Mg. The SFEs calculated for the 34 binary magnesium alloys with the dopant concentration of $\sim 2.08 \%$ are given in Table II. The SFE obtained for the pure magnesium is $21.547 \mathrm{~mJ} / \mathrm{m}^{2}$, which is in agreement with the previous studies.[13, 17, 28] All the doping elements other than $\mathrm{Zn}$ exhibit a reduction in the SFE on alloying with magnesium. The anomalous behavior of $\mathrm{Zn}$ is in contrast to the results of Zhang et al. where all the 34 dopants showed a decrease in the SFE.[17] For a $2.08 \%$ doping concentration, our results show that SFE for $\mathrm{Zn}$-doped $\mathrm{Mg}$ is $1.6 \mathrm{~mJ} / \mathrm{m}^{2}$ larger than that of pure $\mathrm{Mg}$. On the other hand, SFEs of Zn alloys with $1.1 \%$ and $2.5 \% \mathrm{Zn}$ concentrations are more stable than pure $\mathrm{Mg}$ by $0.2 \mathrm{~mJ} / \mathrm{m}^{2}$ and $2.3 \mathrm{~mJ} / \mathrm{m}^{2}$, respectively, according to Zhang et al.[17]. One cannot draw a conclusion on the ability of $\mathrm{Zn}$ to promote stacking faults based on such small changes in SFE, especially when we account for the inherent DFT inaccuracies and the spurious dopantdopant interactions of our model. Therefore, Zn was not disregard for subsequent analyses, especially because $\mathrm{Zn}$ is present in numerous commercial $\mathrm{Mg}$ alloys. 
TABLE II. Calculated SFE and formation energies of the binary Mg alloys with the dopant in bulk, at stacking fault and on surfaces.

\begin{tabular}{|c|c|c|c|c|c|c|}
\hline System & $\operatorname{SFE}\left(\mathrm{mJ} / \mathrm{m}^{2}\right)$ & $E_{f}^{\text {bulk }}(\mathrm{eV})$ & $E_{f}^{I_{1} \mathrm{SF}}(\mathrm{eV})$ & $E_{f}^{(0001)}(\mathrm{eV})$ & $E_{f}^{(10 \overline{1} 1)}(\mathrm{eV})$ & $E_{f}^{(10 \overline{1} 0)}(\mathrm{eV})$ \\
\hline $\mathrm{Al}$ & 14.083 & 0.048 & 0.031 & 0.096 & 0.094 & 0.112 \\
\hline $\mathrm{Ba}$ & 4.316 & 1.311 & 1.273 & -0.343 & -0.612 & -0.580 \\
\hline $\mathrm{Bi}$ & -5.679 & -0.245 & -0.305 & -0.779 & -0.879 & -0.909 \\
\hline $\mathrm{Ca}$ & 14.084 & 0.126 & 0.109 & -0.221 & -0.330 & -0.273 \\
\hline $\mathrm{Ce}$ & 13.402 & 0.167 & 0.150 & 0.036 & 0.114 & 0.125 \\
\hline Cs & -2.044 & 2.833 & 2.781 & -0.410 & -0.573 & -0.639 \\
\hline Dy & 18.854 & -0.010 & -0.016 & 0.280 & 0.336 & 0.473 \\
\hline Er & 19.081 & 0.001 & -0.004 & 0.334 & 0.400 & 0.533 \\
\hline $\mathrm{Eu}$ & 6.815 & 0.411 & 0.379 & -0.209 & -0.387 & -0.310 \\
\hline $\mathrm{Ga}$ & 15.219 & -0.169 & -0.183 & -0.158 & -0.227 & -0.219 \\
\hline $\mathrm{Gd}$ & 18.400 & -0.016 & -0.023 & 0.200 & 0.254 & 0.393 \\
\hline Hf & 11.358 & 0.605 & 0.582 & 1.435 & 1.813 & 1.795 \\
\hline Ho & 19.081 & -0.007 & -0.013 & 0.307 & 0.369 & 0.504 \\
\hline In & 11.358 & -0.367 & -0.399 & -0.464 & -0.510 & -0.501 \\
\hline $\mathrm{K}$ & 5.452 & 1.593 & 1.558 & -0.229 & -0.380 & -0.430 \\
\hline $\mathrm{La}$ & 14.311 & 0.310 & 0.294 & 0.056 & -0.028 & 0.112 \\
\hline $\mathrm{Lu}$ & 18.627 & 0.024 & 0.018 & 0.390 & 0.485 & 0.612 \\
\hline $\mathrm{Na}$ & 12.948 & 0.437 & 0.419 & -0.072 & -0.177 & -0.218 \\
\hline $\mathrm{Nd}$ & 15.447 & 0.080 & 0.067 & 0.109 & 0.096 & 0.238 \\
\hline $\mathrm{Pb}$ & 3.407 & -0.209 & -0.249 & -0.545 & -0.624 & -0.638 \\
\hline $\operatorname{Pr}$ & 14.538 & 0.092 & 0.077 & 0.050 & 0.019 & 0.161 \\
\hline $\mathrm{Rb}$ & 1.590 & 1.492 & 1.448 & -1.047 & -1.205 & -1.256 \\
\hline $\mathrm{Sc}$ & 18.172 & -0.111 & -0.118 & 0.329 & 0.503 & 0.523 \\
\hline $\mathrm{Sm}$ & 17.037 & 0.031 & 0.021 & 0.170 & 0.186 & 0.327 \\
\hline $\mathrm{Sn}$ & 3.862 & -0.260 & -0.299 & -0.467 & -0.531 & -0.538 \\
\hline $\mathrm{Sr}$ & 7.042 & 0.718 & 0.686 & -0.217 & -0.431 & -0.373 \\
\hline $\mathrm{Tb}$ & 18.627 & -0.006 & -0.013 & 0.256 & 0.305 & 0.443 \\
\hline $\mathrm{Ti}$ & 13.175 & 0.649 & 0.630 & 1.213 & 1.479 & 1.429 \\
\hline $\mathrm{Tl}$ & 11.131 & -0.049 & -0.072 & -0.305 & -0.367 & -0.382 \\
\hline $\mathrm{Tm}$ & 19.081 & 0.012 & 0.006 & 0.360 & 0.428 & 0.559 \\
\hline $\mathrm{Y}$ & 18.854 & -0.092 & -0.098 & 0.173 & 0.253 & 0.377 \\
\hline $\mathrm{Yb}$ & 11.358 & 0.282 & 0.260 & -0.079 & -0.220 & -0.150 \\
\hline $\mathrm{Zn}$ & 23.170 & -0.033 & -0.030 & -0.015 & -0.067 & -0.080 \\
\hline $\mathrm{Zr}$ & 11.131 & 0.192 & 0.169 & 0.999 & 1.376 & 1.354 \\
\hline $\mathrm{Mg}$ & 21.547 & - & - & - & - & - \\
\hline
\end{tabular}

\section{B. Wulff plot analysis}

The surface energies calculated for 12 surfaces with the lowest Miller indices $\mathrm{Mg}$ are given in Table III. The equilibrium shape of $\mathrm{Mg}$ crystal is obtained using the Wulff plot construction using these surfaces energies as inputs. The computed Wulff shape is shown in Fig. 3a. It can be seen from the Wulff shape that (0001), (10̄10) and (1011) surfaces are the only surfaces present in $\mathrm{Mg}$ at equilibrium conditions. The area fraction of each surfaces derived from the Wulff shape is given in Table III. Even though (0001) surface is the most energetically favorable surface, most area of the equilibrium magnesium surface

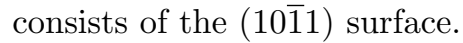

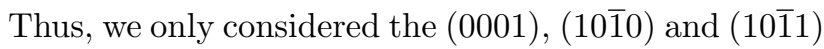
surfaces for our study of the propensity of a dopant to migrate to the surface. Again, for the sake of consistency, the formation energies with the dopants at the surfaces were calculated using 96-atoms slab structures with one
TABLE III. Surface energies and area fraction of 12 surfaces with the lowest Miller indices.

\begin{tabular}{ccc}
\hline \hline Surface & Surface energy $\left(\mathrm{J} / \mathrm{m}^{2}\right)$ & Area fraction \\
\hline$(0001)$ & 0.59 & 0.22 \\
$(10 \overline{1} 0)$ & 0.63 & 0.34 \\
$(10 \overline{1} 1)$ & 0.68 & 0.44 \\
$(10 \overline{1} 2)$ & 0.75 & 0.00 \\
$(11 \overline{2} 0)$ & 0.76 & 0.00 \\
$(11 \overline{2} 1)$ & 0.80 & 0.00 \\
$(20 \overline{2} 1)$ & 0.82 & 0.00 \\
$(21 \overline{3} 0)$ & 0.75 & 0.00 \\
$(21 \overline{3} 1)$ & 0.77 & 0.00 \\
$(2 \overline{1} 12)$ & 0.80 & 0.00 \\
$(21 \overline{3} 2)$ & 0.80 & 0.00 \\
$(22 \overline{4} 1)$ & 0.80 & 0.00 \\
\hline \hline
\end{tabular}

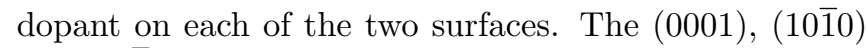

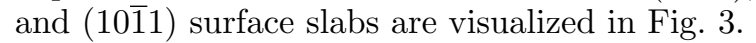




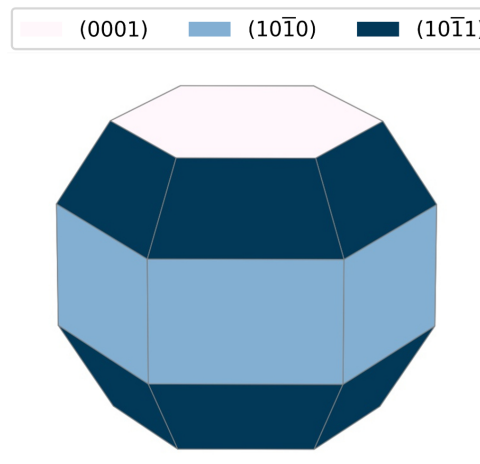

a) Wulff plot

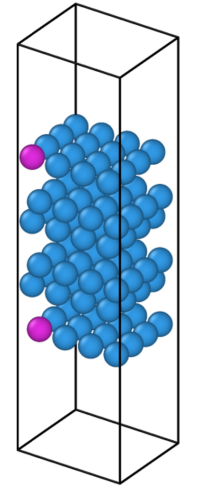

b) (0001) Surface

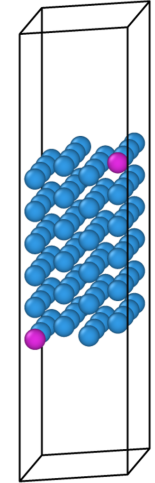

c) $(10 \overline{1} 0)$ Surface

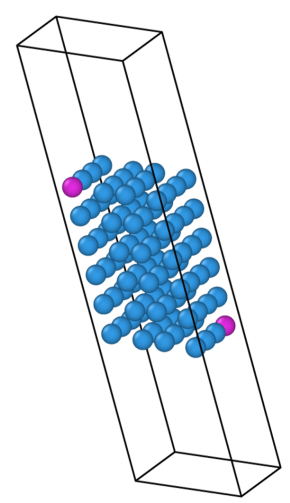

d) (10̄11) Surface

FIG. 3. a) Wulff plot of the equilibrium surfaces of Mg. Slab structures used in the surface calculations. b) (0001) surface, c)

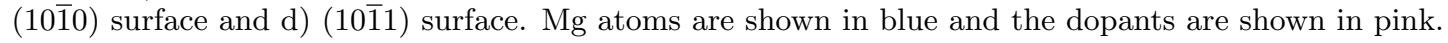

\section{Stability analysis}

The formation energy of the alloy with the dopant atom in the bulk, $E_{f}^{\text {bulk }}$, or at the stacking fault, $E_{f}^{I_{1} \mathrm{SF}}$, should be negative in order to form a stable alloy. As shown in Table II, only 14 out of the 34 considered dopants fulfill that condition: Bi, Dy, Er, Ga, Gd, Ho, $\mathrm{In}, \mathrm{Pb}, \mathrm{Sc}, \mathrm{Sn}, \mathrm{Tb}, \mathrm{Tl}, \mathrm{Y}$ and $\mathrm{Zn}$. It is worth noting that $E_{f}^{I_{1} \mathrm{SF}}$ is smaller than $E_{f}^{\text {bulk }}$ for all dopants other than $\mathrm{Zn}$, which indicates that these dopant are more favorable to be at stacking fault than in the bulk. Er is special case where it is stable only at the stacking fault - all the other stable dopants are stable both in bulk and at the stacking fault. In addition to the 14 dopants that fulfill our stability condition, some dopants such as Al, Lu, Nd, Pr, Sm and Tm display slightly positive values (i.e., less than $0.1 \mathrm{eV}$ for $E_{f}^{\mathrm{bulk}}$ and $E_{f}^{I_{1} \mathrm{SF}}$. Due to the intrinsic errors in DFT, we took a cautious approach and decided not to exclude these 6 dopants at this stage from the pool of potential dopants that are beneficial for Mg-alloy anodes.

A screening based on the stability criterion allows us to disregard 14 out of the 34 dopant candidates. The disregarded dopants were alkali and alkali-earth metals, whose large atomic size compared to that of $\mathrm{Mg}$ makes them rather unstable, three rare-earth elements (Eu, La and $\mathrm{Yb}$ ), and the elements in group 4 of the periodic table (Ti, Zr and Hf). Regarding elements from group 4, there are experimental reports in the literature confirming their low solubility in Mg.[29-31] One could argue the 14 excluded dopants could be metastable at the low doping level considered in this work due to kinetic considerations (e.g., high migration barriers), and they may not segregate to form a separate phase. However, under the operating conditions of the battery where $\mathrm{Mg}$ atoms are continuously plated/stripped from the anode, atomic migration is strongly favored, and thus the segregation of metastable dopants would eventually occur.
It is worth to mention explicitly the case of $\mathrm{Ca}$, which was included in the study by Mandai and Somekawa. The $\mathrm{Mg}-0.3 \mathrm{wt} \% \mathrm{Ca}$ showed a reduction of the overpotential relative to pure $\mathrm{Mg}$.[11] Our calculation show that $\mathrm{Ca}$ and $\mathrm{Mg}$ should segregate, which agrees well with experiments.[32] Mandai and Somekawa speculated that the low amounts of $\mathrm{Ca}$ dopant in their samples dissolve during the first cycles, producing defects that may function as active sites.

\section{Relative energy analysis}

As a final step of the analysis and screening, we evaluated the propensity of the 20 stable dopants to reside in bulk or at stacking fault than migrate to the (0001), $(10 \overline{1} 0)$ or $(10 \overline{1} 1) \mathrm{Mg}$ surfaces. While using the surface at which the dopant is most stable as a reference, we evaluate the relative energies of the dopants in the bulk and at the stacking fault versus on the surface, which are respectively represented as $\Delta E^{\text {bulk }}$ and $\Delta E^{I_{1} \mathrm{SF}}$. The results are shown in Fig. 4.

Among the stable magnesium alloys, $\mathrm{Al}$ and most of the rare earth elements (Dy, Er, Gd, Ho, Lu, Nd, Sc, Sm, $\mathrm{Tb}, \mathrm{Tm}$ and $\mathrm{Y}$ ) have negative $\Delta E^{\text {bulk }}$ and $\Delta E^{I_{1} \mathrm{SF}}$. The negative relative energies imply that these dopants are not prone to migrating to the surface, and thus they are electrochemically inert. In contrast, $\mathrm{Bi}, \mathrm{Pb}, \mathrm{Sn}$, In and $\mathrm{Tl}$ strongly prefer to migrate to the $\mathrm{Mg}$ surface, which will interfere in the stripping (deposition) of $\mathrm{Mg}$ during the discharge (charge) of the battery and potentially react with the electrolyte. Experiments have shown increased overpotentials for $\mathrm{Bi}$ and $\mathrm{Pb}$ dopants with respect to pure Mg.[11, 15]

It should be pointed out that the propensity of the dopants to migrate to the surface depends on the considered surface termination. For example, $\mathrm{Zn}$ and Ga have slightly positive $\Delta E^{\text {bulk }}$ and $\Delta E^{I_{1} S F}$ values because they 


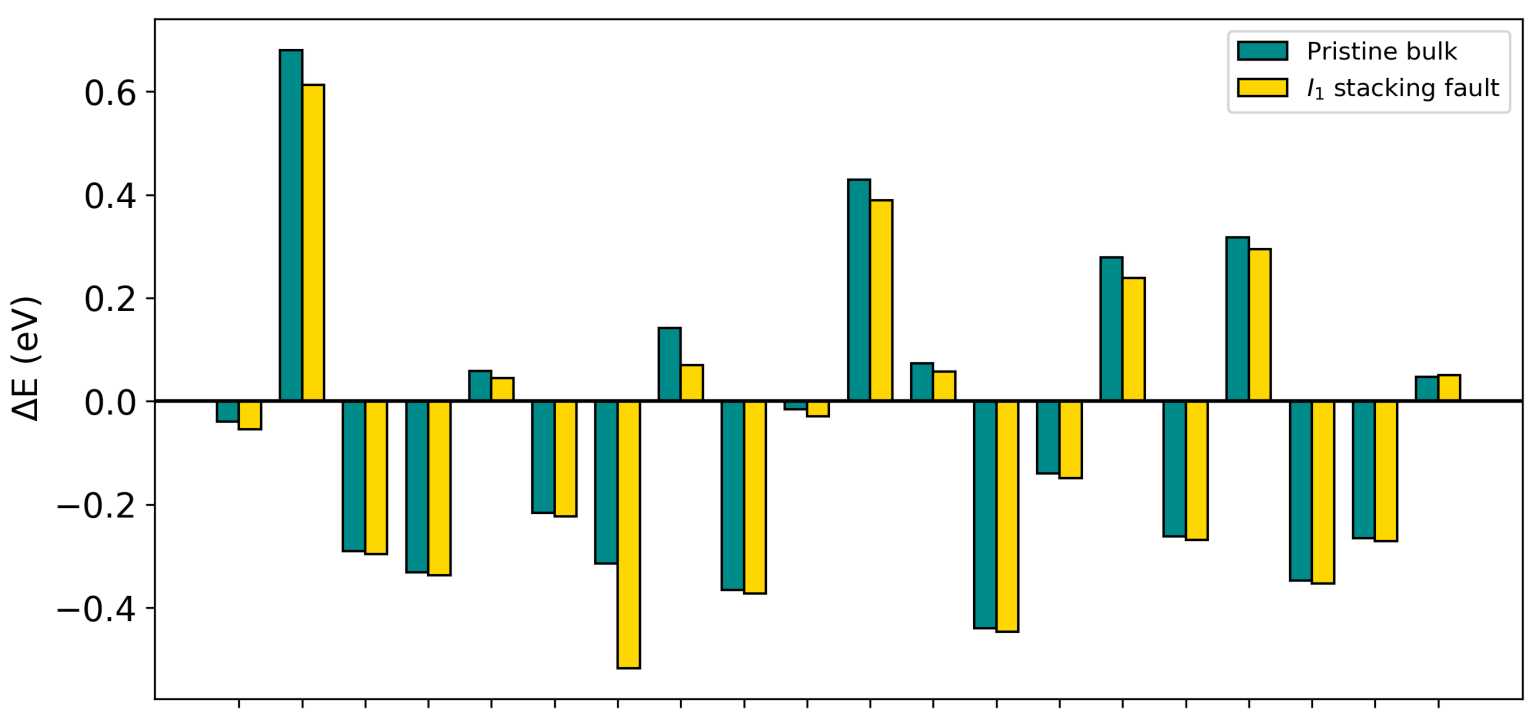

Al Bi Dy Er Ga Gd Ho In Lu Nd Pb Pr Sc Sm Sn Tb Tl Tm Y Zn

FIG. 4. Relative energies of stable Mg alloys.

energetically prefer to be on the $(10 \overline{10})$ or $(10 \overline{1} 1)$ surfaces. However, they are more stable to be in the bulk or at the $I_{1} \mathrm{SF}$ compared to the (0001) surface. Our results show a good agreement with the experimental results even for the borderline cases where the magnitudes of $\Delta E^{\text {bulk }}$ and $\Delta E^{I_{1} \mathrm{SF}}$ are small. The study of Schloffer et al. on the electrochemical behavior of Mg-1.63 wt\% Zn in APC electrolyte reported that the alloy exhibits a high propensity to be passivated,[16] indicating that $\mathrm{Zn}$ participates in surface processes. Along the same lines, Mandai and Somekawa,[11] Zhao et al.[14] and Wang et al.[15] reported an increased overpotential in the AZ31 alloy, containing $\mathrm{Zn}$, with respect to pure $\mathrm{Mg}$. Schloffer et al. also showed that the addition of $1.55 \mathrm{wt} \% \mathrm{Gd}$ does not have any adverse effect on the anode performance.[16] These experimental observations are in a good agreement with our findings where Gd does not migrate to Mg surfaces while $\mathrm{Zn}$ does.

Based on the identified dopants, the commercial alloy WE43 with a composition of Mg-4 wt\% Y-2.25 wt\% Nd$0.15 \mathrm{wt} \% \mathrm{Zr}$ will be a suitable candidate as magnesium alloy anode. The two main dopants in WE43, Y and Nd, are both stable in the bulk, promote stacking faults and do not migrate to the surface. $\mathrm{Zr}$ is added to magnesium alloys as a grain refiner although it has limited solubility in magnesium; the undissolved $\mathrm{Zr}$ act as a nucleation site during solidification and form fine-equiaxed grains. [33] In contrast, the alloys in the AZ family (the most widely used type of commercial $\mathrm{Mg}$ alloys) contain $\mathrm{Al}$ and $\mathrm{Zn}$, are expected to be unsuitable for $\mathrm{Mg}$ battery anodes as $\mathrm{Zn}$ tends to migrate to the surface.

\section{CONCLUSION}

We performed a theoretical study of 34 dopants reported in the literature as beneficial to improve magnesium ductility to explore their potential use as anodes in magnesium batteries. We started by checking that the studied alloys at the doping level considered in this work $(\sim 2.08 \%)$ promote stacking faults defects, whose presence enhances magnesium ductility. Subsequently, we examined whether the dopants tend to segregate and form separate phases or prefer to remain in the alloy. We found that only 20 out of the 34 dopants, mostly rareearth elements, are stable in the alloy. Finally, we investigated these 20 stable dopants and their propensity to migrate to the alloy's surface. We considered that those dopants that migrate to the surface could interfere with the electrochemical process (they could, for instance, produce passivation or degrade the electrolyte), and thus we disregarded them as useful dopants. Within this sequential analysis, we identified 12 potential favorable dopants: Al, Dy, Er, Gd, Ho, Lu, Nd, Sc, Sm, Tb, Tm and Y. Remarkably, the commercial alloy WE43 contains Y and $\mathrm{Nd}$, both included in our shortlist of beneficial dopants, so we encourage experimental tests with this alloy.

\section{ACKNOWLEDGEMENT}

The authors thank Piotr Jankowski for fruitful discussions. The authors wish to acknowledge support from the "European Magnesium Interactive Battery Community (e-Magic)" FET-Proactive project (Contract N. 
824066). JMGL acknowledges support from the Villum Foundation's Young Investigator Programme (4th round, project: In silico design of efficient materials for next generation batteries. Grant number: 10096).

\section{REFERENCES}

[1] R. Mohtadi, F. Mizuno, Beilstein J. Nanotechnol. 2014, 5, 1291-1311.

[2] T. S. Arthur, N. Singh, M. Matsui, Electrochem. Commun. 2012, 16, 103-106.

[3] N. Singh, T. S. Arthur, C. Ling, M. Matsui, F. Mizuno, Chem. Commun. 2013, 49, 149-151.

[4] F. Murgia, E. T. Weldekidan, L. Stievano, L. Monconduit, R. Berthelot, Electrochem. Commun. 2015, 60, 56-59.

[5] K. Periyapperuma, T. T. Tran, M. Purcell, M. Obrovac, Electrochim. Acta 2015, 165, 162-165.

[6] J. Niu, Z. Zhang, D. Aurbach, Adv. Energy Mater. 2020, 10, 2000697.

[7] D. Aurbach, Z. Lu, A. Schechter, Y. Gofer, H. Gizbar, R. Turgeman, Y. Cohen, M. Moshkovich, E. Levi, Nature 2000, 407, 724-727.

[8] O. Mizrahi, N. Amir, E. Pollak, O. Chusid, V. Marks, H. Gottlieb, L. Larush, E. Zinigrad, D. Aurbach, J. Electrochem. Soc. 2008, 155, A103.

[9] C. J. Barile, E. C. Barile, K. R. Zavadil, R. G. Nuzzo, A. A. Gewirth, J. Phys. Chem. C 2014, 118, 27623-27630.

[10] R. Mohtadi, M. Matsui, T. S. Arthur, S.-J. Hwang, Angew. Chem. 2012, 124, 9918-9921.

[11] T. Mandai, H. Somekawa, Chem. Commun. 2020, $56,12122-12125$.

[12] S. Sandlöbes, M. Friák, S. Korte-Kerzel, Z. Pei, J. Neugebauer, D. Raabe, Sci Rep 2017, 7, 1-8.

[13] S. Sandlöbes, M. Friák, S. Zaefferer, A. Dick, S. Yi, D. Letzig, Z. Pei, L.-F. Zhu, J. Neugebauer, D. Raabe, Acta Mater. 2012, 60, 3011-3021.
[14] H. Zhao, P. Bian, D. Ju, J. Environ. Sci. 2009, 21, S88-S91.

[15] N. Wang, R. Wang, C. Peng, Y. Feng, B. Chen, Corros. Sci. 2012, 64, 17-27.

[16] D. Schloffer, S. Bozorgi, P. Sherstnev, C. Lenardt, B. Gollas, J. Power Sources 2017, 367, 138-144.

[17] J. Zhang, Y. Dou, G. Liu, Z. Guo, Comput. Mater. Sci. 2013, 79, 564-569.

[18] Z. Pei, M. Friák, S. Sandlöbes, R. Nazarov, B. Svendsen, D. Raabe, J. Neugebauer, New J. Phys. 2015, 17, 093009.

[19] R. Mises, Z. Angew. Math. Mech. 1928, 8, 161-185.

[20] M. Yoo, Metall. Trans. A 1981, 12, 409-418.

[21] Z. Wu, W. Curtin, Nature 2015, 526, 62-67.

[22] G. Kresse, J. Furthmüller, Phys. Rev. B 1996, 54, 11169-11186.

[23] G. Kresse, J. Furthmüller, Comput. Mater. Sci. 1996, $6,15-50$.

[24] J. P. Perdew, K. Burke, M. Ernzerhof, Phys. Rev. Lett. 1996, 77, 3865-3868.

[25] P. Blöchl, Phys. Rev. B 1994, 50, 17953-17979.

[26] R. Tran, Z. Xu, B. Radhakrishnan, D. Winston, W. Sun, K. A. Persson, S. P. Ong, Sci. Data 2016, 3, $1-13$.

[27] S. P. Ong, W. D. Richards, A. Jain, G. Hautier, M. Kocher, S. Cholia, D. Gunter, V. L. Chevrier, K. A. Persson, G. Ceder, Comput. Mater. Sci. 2013, 68, 314-319.

[28] Y. Wang, L.-Q. Chen, Z.-K. Liu, S. Mathaudhu, Scr. Mater. 2010, 62, 646-649.

[29] M. Qian, D. StJohn, M. Frost, Scr. Mater. 2002, 46, 649-654.

[30] J. Murray, Bull. Alloy Phase Diagramsms 1986, 7, $245-248$.

[31] J. Schaum, H. Burnett, J. Res. Natl. Bur. Stand 1952, 49, 155 .

[32] R. Agarwal, J. J. Lee, H. L. Lukas, F. Sommer, Zeitschrift für Metallkunde 1995, 86, 103-108.

[33] Y. Ding, C. Wen, P. Hodgson, Y. Li, J. Mater. Chem. B 2014, 2, 1912-1933. 


\section{GRAPHICAL ABSTRACT}
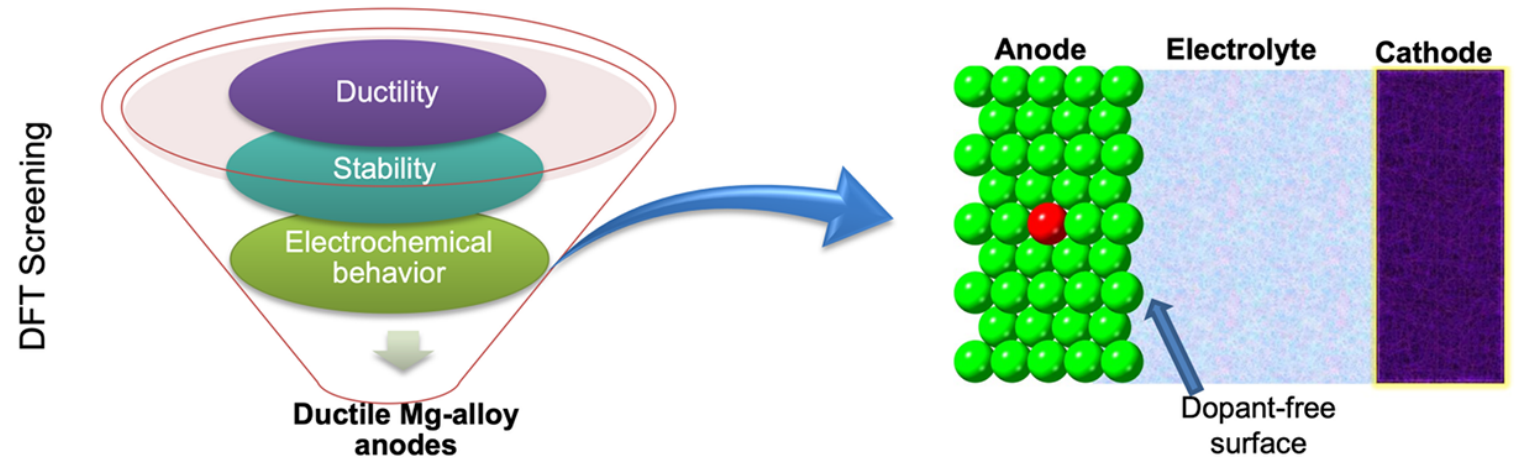

We performed a computational screening of dopants that can alloy with magnesium and use as an anode in magnesium batteries. Three properties were evaluated for this screening: ductility improvement, stability of the alloy and propensity of dopant to reside in bulk to avoid electrochemical reactions. We considered 34 dopants and identified 12 dopants that are suitable for magnesium battery applications.

Keywords: Density functional calculations, Magnesium batteries, Alloys, Magnesium alloy anodes 